\title{
Identificazione e caratterizzazione dei determinanti genetici di antibiotico-resistenza in ceppi di Salmonella enterica di origine animale
}

\section{Cristina Pezzella', Laura Villa', Alessia Bertini', Antonia Ricci², Elisabetta Di Giannatale², Ida Luzzi' e Alessandra Carattoli'}

IIstituto Superiore di Sanità-Dipartimento di Malattie Infettive, Parassitarie ed Immunomediate.

${ }^{2}$ Istituto Zooprofilattico delle Venezie.

${ }^{3}$ Istituto Zooprofilattico dell'Abruzzo e del Molise.

Key words: Salmonella Enterica, resistence, genetic determinants

\section{SUMMARY}

Tetracyclines are broad-spectrum agents, exhibiting activity against a wide range of gram-positive and gram-negative bacteria and are currently used for therapy and prophylaxis of human infections and for the prevention and control of bacterial infections in veterinary medicine. Streptomycin has only limited current usage in clinical medicine, but this antibiotic remains important for therapeutic and growth promotion in animals and for the bacterial disease control in plants. The increasing incidence of resistance to streptomycin and tetracyclines has been reported worldwide in Salmonella spp. of human and animal origin.

Fifty-eight multidrug-resistant Salmonella enterica strains of twenty different serotypes, were chosen among the collection of multidrug-resistant strains isolated from animals and food of animal origin at the Istituto Zooprofilattico Sperimentale delle Venezie and at the Istituto Zooprofilattico Sperimentale dell'Abruzzo e Molise, during their routine surveillance activity in the 2000 and $200 \mathrm{I}$ period. All strains showed resistance to at least three different antimicrobials: tetracycline and streptomycin resistances represent the $98 \%$ and $95 \%$ of the strains, respectively. Salmonella isolates were analyzed for the presence of genetic determinants conferring streptomycin and tetracyclines resistance by PCR for the tet(A) and strA-strB genes, respectively. The strA-strB genes were highly prevalent in Salmonella strains of our collection, being detected in the $83 \%$ of the streptomycin resistant strains; the $68 \%$ of the tetracycline resistant strains were tet(A) gene positive, indicating that this gene is widely diffused in Salmonella strains circulating in animals in Italy.

Two prevalent repN- and repll-resistance plasmids were identified in Salmonella isolates of our collection. In many strains, the strA-strB genes were linked to a particular Tn5393-derivative transposon, characterized by the presence of the insertion sequence IS/ I33. The IS/ /33-variant was previously described in Erwinia amylovora strains from plant sources. The identification of the Tn5393::IS / I33 element in Salmonella suggests novel scenarios of resistance transmission among zoonotic and plant pathogens.

\section{INTRODUZIONE}

I dati di sorveglianza raccolti in questi ultimi anni in Italia e in Europa mostrano un preoccupante aumento delle resistenze agli antibiotici nei vari sierotipi di Salmonella enterica. In particolare è in costante incremento in tutti i sierotipi, la resistenza ad antibiotici quali le tetracicline e la streptomicina $(2,20)$. Le tetracicline oltre ad essere utilizzate nel trattamento di infezioni umane sostenute da batteri Gram positivi e Gram negativi, sono ampiamente usate in campo veterinario per la prevenzione ed il controllo di infezioni batteriche in zootecnia e in agricoltura (3). La streptomicina ha un uso terapeutico limitato nell'uomo, ma è importante come promotore di crescita e viene utilizzata come agente terapeutico negli animali e per il controllo delle malattie batteriche nelle piante (14).

I geni più diffusi che conferiscono la resistenza alle tetracicline nei batteri Gram negativi sono $\mathrm{i}$ geni $\operatorname{tet}(\mathrm{A}), \operatorname{tet}(\mathrm{B})$ e $\operatorname{tet}(\mathrm{G})(6)$. La resistenza alla streptomicina è stata descritta frequentemente associata alla presenza dei geni $\operatorname{str} A-s t r B$, spesso veicolati da piccoli plasmidi non coniugativi (15). I geni che conferiscono la resistenza ad altri aminoglicosidi sono invece più spesso localizzati in elementi genetici noti come integroni (7), ampiamente diffusi nelle Enterobacteriaceae e in Pseudomonas. Questi elementi hanno la capacità di acquisire cassette geniche e di integrarle sotto il controllo di un'unità trascrizionale in grado di promuoverne l'espressione (8).

Lo scopo di questo lavoro è stato quello di identificare i geni di resistenza, in particolare quelli che conferiscono la resistenza alla streptomicina ed alle tetracicline e gli integroni, in ceppi multiresistenti di $S$. enterica isolati in Italia da animali d'allevamento e da alimenti di origine animale. 
Sono stati inoltre analizzati i meccanismi genetici responsabili della diffusione di queste resistenze fra i diversi sierotipi, caratterizzando i plasmidi di resistenza.

\section{MATERIALI E METODI \\ Ceppi di Salmonella}

Cinquantotto ceppi di S. enterica non correlati epidemiologicamente, sono stati scelti fra un totale di 835 stipiti multiresistenti isolati presso 1'Istituto Zooprofilattico Sperimentale (IZS) delle Venezie (IZS-VE) e l'IZS dell'Abruzzo e Molise (IZS-AM) negli anni 2000 e 2001 da animali e da alimenti di origine animale destinati all'alimentazione umana. I ceppi studiati appartenevano a 20 sierotipi differenti, di cui i sierotipi Hadar (12 ceppi), Blockley ed Heidelberg, (6 ceppi per ciascun sierotipo), Derby (5 ceppi) e Typhimurium (4 ceppi) erano più rappresentati; i rimanenti sierotipi (Agona, Anatum, Bredeney, Colorado, Enteritidis, Give, Infantis, Kisii, Livingstone, London, Panama, Saintpaul, Senftenberg, Tshiongwe e Virkow) rappresentavano ciascuno meno del 5\% del totale dei ceppi. I ceppi sono stati scelti tra quelli che presentavano la resistenza ad almeno tre antibiotici (aminoglicosidi, tetracicline, trimethoprim e $\beta$-lattamici) e che provenivano da fonti animali diverse. Tutti i ceppi di Salmonella erano stati isolati, identificati biochimicamente e sierologicamente secondo procedure standardizzate (10). La sensibilità agli antibiotici era stata determinata mediante il metodo di diffusione in agar (12). Il pannello di antibiotici saggiati comprendeva colistina $(10 \mu \mathrm{g})$, acido nalidixico $(30 \mu \mathrm{g})$, ampicillina $(10 \mu \mathrm{g})$, cefotaxime $(30 \mu \mathrm{g})$, ciprofloxacina $(5 \mu \mathrm{g})$, enrofloxacina $(5 \mu \mathrm{g})$, cloramfenicolo $(30 \mu \mathrm{g})$, gentamicina $(10 \mu \mathrm{g})$, kanamicina $(30 \mu \mathrm{g})$, streptomicina $(10 \mu \mathrm{g})$, sulfonamidi $(300 \mu \mathrm{g})$, tetraciclina $(30 \mu \mathrm{g})$, trimethoprim-sulfametoxazolo $(1.25 / 23.75 \mu \mathrm{g})$, amoxicillina-acido clavulanico $(30 \mu \mathrm{g})$, cefalotina $(30 \mu \mathrm{g})$ e neomicina $(30 \mu \mathrm{g})$.

\section{Coniugazione batterica}

Per dimostrare il trasferimento dei geni di resistenza sono stati eseguiti esperimenti di coniuga- zione usando come cellula ricevente il ceppo mutante Escherichia coli $\mathrm{K}-12 \mathrm{CSH} 26$ resistente alla rifampicina o all'acido nalidixico (11). I transconiuganti sono stati selezionati su piastre di Luria Bertani contenenti $100 \mu \mathrm{g} / \mathrm{ml}$ di rifampicina (o $40 \mu \mathrm{g} / \mathrm{ml} \mathrm{di} \mathrm{acido} \mathrm{nalidixico)} \mathrm{e} 30 \mu \mathrm{g} / \mathrm{ml} \mathrm{di}$ streptomicina o $30 \mu \mathrm{g} / \mathrm{ml}$ di tetraciclina o, infine, $50 \mu \mathrm{g} / \mathrm{ml}$ di ampicillina. Sui transconiuganti ottenuti è stato eseguito un antibiogramma per verificare quali resistenze fossero state trasferite.

\section{Studio di geni di resistenza e integroni}

L'estrazione del DNA totale è stata effettuata secondo il metodo di Ezaki (5), mentre il DNA plasmidico ad alto peso molecolare è stato estratto mediante il sistema di purificazione Concert ${ }^{\mathrm{TM}}$ High Purity Plasmid (Life Technologies, 1).

Le reazioni di PCR sono state effettuate preparando una miscela di reazione contenente, in $25 \mu \mathrm{ldi}$ volume finale, $\mathrm{i}$ seguenti reagenti: $300 \mathrm{ng}$ di DNA, $200 \mu \mathrm{M}$ di dNTP, $1 \mu \mathrm{M}$ di ogni coppia di oligonucleotidi, 1x PCR buffer e 1.25U di Taq DNA-polimerasi (Roche Diagnostic). Per la ricerca del gene tet $(A)$ sono stati utilizzati gli oligonucleotidi TetAF (5'-GTAATTCTGAGCACTGT3') e TetAR (5'-CCTGGACAACATTGCTT-3'), per i geni $\operatorname{str} A-s t r B$ sono stati utilizzati strAF (5'AGCAGAGCGCGCCTTCGCTG-3') e strBR (5'-CCAAAGCCCACTTCACCGAC-3').

Le cassette geniche localizzate negli integroni sono state amplificate con la coppia di oligonucleotidi 5'CS (5'-GGCATCCAAGCAGCAAG3') e 3'CS (5'-AAGCAGACTTGACCTGA-3'). La ricerca per PCR dei gruppi di incompatibilità IncI e IncN è stata eseguita mediante l'uso degli oligo I1 (5'-CGGGACAGGATGTGCAA-3'), I2 (5'-ACTTCAGGCTCCTTACGGG-3'), N1 (5'AGTTCACCACCTACTCGCTCCG-3') e N2 (5'-CAAGTTCTTCTGTTGGGATTCCG-3'), rispettivamente. L'analisi per PCR della presenza del trasposone Tn5393::IS1133 è stata eseguita con gli oligo smAR (5'-TCCTCCTGCCAGTTGATCAC-3') e IS1133F (5'-GATTGGCTGGGCAACAGGTGA-3'). Gli ampliconi sono stati purificati con il Qiagen PCR Purification kit (Qiagen, Milano, Italia), e sequenziati. Le sequen-

Tabella I. Caratteristiche genetiche delle Salmonelle analizzate in questo studio

\begin{tabular}{|c|c|c|c|}
\hline Caratteristiche genetiche $^{a}$ & IZS-VE $n=36$ & IZS-AM n=22 & Totali $n=58$ \\
\hline $\operatorname{tet}(\mathrm{A})+/ \mathrm{Te}^{\mathrm{R}}$ & $24 / 35(68.5 \%)$ & $14 / 22(63.6 \%)$ & $38 / 57(68.0 \%)$ \\
\hline strA-strB+ / Sm ${ }^{R}$ & $32 / 35(91.4 \%)$ & I4/20 (70.0\%) & $46 / 55(83.0 \%)$ \\
\hline Integroni & $\mathrm{I} / / 36(30.5 \%)$ & $6 / 22(27.2 \%)$ & $17 / 58(29.0 \%)$ \\
\hline Plasmidi IncN & $13 / 36(36.0 \%)$ & $2 / 22(9.0 \%)$ & $15 / 58(25.8 \%)$ \\
\hline Plasmidi Incl & $6 / 36(16.6 \%)$ & $2 / 22(9.0 \%)$ & $8 / 58(13.8 \%)$ \\
\hline strA+ / IS / /33 & $10 / 32(31.2 \%)$ & $6 / 14(42.8 \%)$ & $16 / 46(34.8 \%)$ \\
\hline
\end{tabular}

${ }^{a^{+}}$: positivo per la presenza del gene; $\mathrm{Te}^{\mathrm{R}}$ : no. di ceppi tetraciclina resistenti; $\mathrm{Sm}^{\mathrm{R}}$; no. ceppi streptomicina resistenti; IS / I33: no. ceppi positivi per il trasposone Tn5393::IS / I33. 
ze sono state eseguite in un sequenziatore automatizzato 373 (Perkin-Elmer, Foster City, Calif.) presso la ditta M-Medical (Pomezia, Italia).

L'analisi delle sequenze nucleotidiche ottenute è stata effettuata con il programma NCBI "BLAST" (Versione 2.0) (www.ncbi.nlm.nih.gov).

\section{Analisi dei plasmidi}

L'analisi dei plasmidi è stata eseguita mediante digestione con l'enzima di restrizione EcoRI. I frammenti di restrizione sono stati separati e visualizzati mediante una corsa elettroforetica in gel d'agarosio allo $0.8 \%$ in tampone TAE $1 \mathrm{x}$ (40 $\mathrm{mM}$ Tris- $\mathrm{HCl}, 0.1 \mathrm{mM}$ EDTA, $\mathrm{pH} 8.0)$, a voltaggio costante di $15 \mathrm{~V} / \mathrm{cm}$ per 14 ore, poi trasferito su membrana di nylon (16).

Gli esperimenti di ibridazione sono stati condotti secondo procedura standard (13). La determinazione dei gruppi d'incompatibilità dei plasmidi è stata eseguita come descritto da Couturier et al. (4), mediante l'uso di sonde specifiche gentilmente fornite dal Prof. W.K. Maas, per i seguenti gruppi d'incompatibilità: FIA, FIB, FII, I1, B/O, $\mathrm{K}, \mathrm{HI} 1, \mathrm{HI} 2, \mathrm{~L} / \mathrm{M}, \mathrm{N}, \mathrm{P}, \mathrm{W}, \mathrm{Y}$ e X.

\section{RISULTATI}

Caratteristiche di resistenza agli antibiotici dei ceppi esaminati

I ceppi studiati mostravano resistenza ad almeno tre differenti antibiotici: 55/58 ceppi (95\%) erano resistenti alla streptomicina, 57/58 (98\%) alla tetraciclina e il $66 \%$ dei ceppi era resistente all'ampicillina. Il $40 \%$ dei ceppi era resistente all'amoxicillina-acido clavulanico. La percentuale di resistenza alla gentamicina era del $26 \%$, mentre quelle alla kanamicina ed alla neomicina rappresentavano rispettivamente il $41 \%$ ed il $29 \%$ del totale dei ceppi. Il 52\% degli stipiti esaminati era resistente ai sulfonamidi, mentre il 33\% dei ceppi erano resistenti al trimethoprim. Le resistenze più diffuse nella collezione studiata riguardavano quindi la streptomicina (S), la tetraciclina (T) e l'ampicillina (A). In particolare, 39 ceppi, pari al $62 \%$ del totale, mostravano un fenotipo di resistenza multipla R-STA.

Ricerca dei geni di resistenza alla streptomicina e tetraciclina e di integroni mediante PCR

Su tutti i ceppi di Salmonella resistenti alla tetraciclina è stata effettuata una PCR con la coppia di oligonucleotidi TAF-TAR, specifica per il gene tet(A). Dei 57 ceppi di Salmonella resistenti alla tetraciclina, 38 isolati $(68 \%)$ sono risultati positivi all'amplificazione del gene tet(A) (Tabella 1). Dei 55 ceppi di Salmonella resistenti alla streptomicina, $46(83 \%)$ hanno mostrato la presenza dei geni strA-strB, mediante PCR con gli oligonucleotidi SAF e SBR (Tabella 1).

Diciassette dei 58 stipiti (29\%) di Salmonella ana- lizzati sono risultati positivi per la presenza di integroni di classe 1. Gli ampliconi ottenuti sono stati completamente sequenziati per determinare il tipo di cassette geniche contenute. I geni più diffusi localizzati sugli integroni sono risultati aadA1 e aadA2, responsabili della resistenza alla streptomicina e spectinomicina, e $d f r A l$, codificante la resistenza al trimethopim.

\section{Analisi dei plasmidi di resistenza}

Allo scopo di verificare la trasmissibilità delle resistenze dei geni $\operatorname{str} A-\operatorname{str} B$ e $\operatorname{tet}(\mathrm{A})$ e degli integroni, sono stati effettuati degli esperimenti di coniugazione batterica di 27 ceppi della collezione. Questi ceppi erano rappresentativi di 15 differenti sierotipi presenti nella collezione, provenienti da varie fonti d'isolamento ed includevano stipiti sia positivi che negativi agli integroni ed ai geni di resistenza $\operatorname{str} A-\operatorname{str} B$ e $\operatorname{tet}(\mathrm{A})$. Sono stati ottenuti 9 transconiuganti per i quali è stata dimostrata una localizzazione plasmidica degli integroni e dei geni tetA e strA-strB.

Al fine di approfondire ulteriormente lo studio dei plasmidi di resistenza coniugativi, sono stati scelti quattro transconiuganti (T27/30, T17/25, T17/1 e T17/19) per l'analisi del profilo di restrizione plasmidico e per la determinazione del gruppo di incompatibilità.

La figura I mostra l'analisi dei frammenti di restrizione per l'enzima $E c o$ RI ed i risultati ottenuti sui plasmidi prototipo mediante ibridazione con sonde specifiche per i geni $\operatorname{str} \mathrm{A}-\operatorname{str} \mathrm{B}, \operatorname{tet}(\mathrm{A}) \mathrm{e}$ per il gene dell'integrasi intIl, associata agli integroni.

Questa analisi ha permesso di localizzare i geni di resistenza e gli integroni in specifiche bande plasmidiche e ha messo in evidenza la presenza di plasmidi con profilo di restrizione simile in ceppi diversi. La caratterizzazione del gruppo di incompatibilità ha permesso di rilevare che i plasmidi contenuti nei transconiuganti T17/1 e T17/19 appartengono al gruppo d'incompatibilità $\mathrm{N}$ e quelli dei ceppi T17/25 e T27/30 al gruppo d'incompatibilità I. Una volta identificati questi due tipi di plasmidi nei transconiuganti prototipo, sono state messe a punto PCR specifiche per i gruppi di incompatibilità I e N allo scopo di identificare plasmidi analoghi eventualmente presenti negli altri ceppi di Salmonella della collezione. Mediante questa analisi si è potuto dimostrare che $13 / 36(36 \%)$ e $6 / 35(20 \%)$ ceppi tra quelli isolati dall'IZS-VE presentavano plasmidi appartenenti al gruppo d'incompatibilità $\mathrm{N}$ e al gruppo d'incompatibilità $\mathrm{I}$, rispettivamente. Plasmidi IncN e IncI sono stati ritrovati anche nella collezione di ceppi dell'IZS-AM seppure rappresentati in misura più ridotta (Tabella 1 ).

Al fine di caratterizzare meglio i plasmidi IncN, i 
più diffusi negli isolati della nostra collezione, sono state clonate e sequenziate le regioni plasmidiche fiancheggianti i geni strA-strB. Questa analisi ha evidenziato la localizzazione dei geni strAstr $B$ nel trasposone Tn5393. In particolare il gene str $A$ è stato trovato associato alla sequenza d'inserzione IS 1133. È stata quindi messa a punto una PCR per studiare la diffusione dell'elemento IS1133 in tutti i ceppi della collezione. Sedici dei 46 ceppi positivi ai geni $\operatorname{str} A-s t r B(35 \%)$ hanno mostrato l'associazione di questi geni alla sequenza di inserzione IS1133. Da notare che nel sierotipo Hadar il trasposone Tn5393::IS1133 era molto rappresentato essendo presente in 9 ceppi su 12.

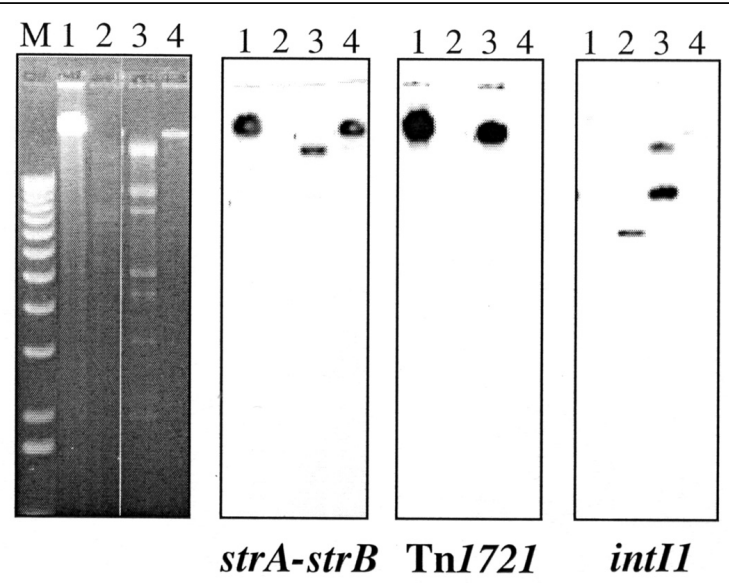

Figura I. Profili plasmidici di restrizione con l'enzima EcoRI dei transconiuganti I7/I, I7/25, 27/30 e I7//9 (pannello A, corsie I, 2, 3 e 4, rispettivamente). Ibridazioni con i geni strA-strB (pannello $B$ ), il gene tet(A) (pannello $C$ ), e l'integrasi intll (pannello D).

\section{DISCUSSIONE}

Dall'analisi molecolare di ceppi multiresistenti di Salmonella si possono ottenere informazioni utili sulla diffusione dei geni di resistenza, sulla loro origine e sui veicoli genetici responsabili della resistenze agli antibiotici. Il dato più significativo che emerge dalla nostra analisi è quello della presenza di geni diffusi e ricorrenti che conferiscono la resistenza alla streptomicina, $\operatorname{str} A-\operatorname{str} B$, e alla tetraciclina, $\operatorname{tet}(\mathrm{A})$. La diffusione di questi geni in ceppi di Salmonella non correlati epidemiologicamente fra loro, appartenenti a sierotipi diversi, isolati da fonti eterogenee di natura animale, nonché provenienti da regioni italiane geograficamente non confinanti, evidenzia quanto sia rilevante il ruolo del trasferimento orizzontale delle resistenze. Infatti alcuni ceppi presentano questi geni localizzati su plasmidi. La determinazione del gruppo di incompatibilità dei plasmidi si è rivelata uno strumento molto efficace per eviden- ziare elementi genetici comuni, presenti in ceppi non correlati. Il gruppo di incompatibilità rappresenta una caratteristica genetica che esprime il grado di similitudine tra plasmidi diversi, indicandone l'appartenenza a famiglie conservate. L'analisi ha messo in evidenza la presenza, nei ceppi prototipo, di plasmidi del tipo IncI ed IncN. La ricerca per PCR di plasmidi dei gruppi IncI e IncN eseguita sull'intera collezione ha rivelato la presenza di molti di questi repliconi nei ceppi provenienti dal nord Italia ma anche, seppure con minore frequenza, in quelli isolati nel centro-sud. Questo dimostra la diffusione e la circolazione di plasmidi comuni in sierotipi diversi di Salmonella della nostra collezione.

L'osservazione che molti dei geni strA-strB erano localizzati nel trasposone Tn5393::IS1133 ha permesso di formulare una interessante ipotesi sulla possibile origine di questo determinante di resistenza in Salmonella. Infatti questa variante di trasposone era stata precedentemente descritta solo in isolati di Erwinia amylovora, un patogeno delle piante $(9,17,18)$. La presenza in Salmonella di trasposoni caratteristici dei patogeni delle piante apre un nuovo scenario sul possibile scambio di geni di resistenza tra batteri che colonizzano ambienti completamente diversi. L'acquisizione di queste resistenze, può essere spiegata dal fatto che Salmonella è un batterio ubiquitario che circola nell'ambiente, negli animali e nell'uomo, e che ha quindi la possibilità di venire in contatto con batteri che colonizzano nicchie ecologiche diverse, anche i patogeni delle piante, e di acquisire mediante scambi orizzontali, plasmidi e altri determinanti genetici.

In conclusione, l'analisi molecolare dei ceppi ha mostrato la presenza di pochi elementi genetici, ma molto diffusi circolanti nei ceppi isolati dagli animali in Italia, indicando quanto sia importante per la diffusione della resistenza agli antibiotici, il trasferimento orizzontale dei geni e gli scambi genetici tra batteri

\section{BIBLIOGRAFIA}

1. Birnboim H, Doly J. A rapid alkaline extraction procedure for screening recombinant plasmid DNA. Nucleic Acid Res 1979; 7: 1513-23

2. Busani L, Graziani C, Battisti A et al. Antibiotic resistance in Salmonella enterica serotypes Typhimurium, Enteritidis and Infantis from human infections, foodstuffs and farm animals in Italy. Epidemiol Infect 2004; 1-7 (in corso di stampa)

3. Chopra I, Roberts M. Tetracicline antibiotics: mode of action, applications, molecular biology and epidemiology of bacterial resistance. Microbiology and Molecular Biology Reviews 2001; 65(2): 232-60

4. Couturier M, Bex F, Bergquist P L, Maas W K. 
Identification and classification of bacterial plasmids. Microbiol Reviews 1988; 52(3): 375-95

5. Ezaki T, Takeuchi N, Liu S L, Kai A, Yamamoto H, Yabuuchi E. Small-scale DNA preparation for rapid genetic identification of Campylobacter species without radioisotope. Microbiol Immunol 1988; 32: 141-50

6. Frech G, Schwarz S. Plasmid-encoded tetracycline resistance in Salmonella enterica subsp. enterica serovars cholerasuis and typhimurium: identification of complete and truncated Tn1721 elemnts. FEMS Microbiol Lett 1999; 176(1): 97-103

7. Grinsted J, de la Cruz F, Schmitt R. The Tn21 subgroup of bacterial transposable elements. Plasmid 1990; 24: 163-89

8. Hall RM, Collis CM. Mobile gene cassettes and integrons: capture and spread of genes by site-specific recombination. Molecular Microbiology 1995; 15: 593-600

9. L'Abee-Lund TM, Sorum H. Functional Tn5393-like transposon in the $\mathrm{R}$ plasmid pRAS2 from the fish pathogen Aeromonas salmonicida subspecies salmonicida isolated in Norwey. Appl Environ Microbiol 2000; 66: 5533-35

10. Lennette EH, Balows A, Hausler Jr WJ, Shadomy H J. Enterobacteriaceae. In: Manual of Clinical Microbiol. Fourth Ed. ASM Eds, American Society for Microbiology, Washington DC 1985; 263-77.

11. Maimone F, Colonna B, Bazzicalupo P, Oliva B, Nicoletti M, Casalino M. Plasmids and transposable elements involved in Salmonella wien. J Bacteriol 1979; 193: 369-75

12. National Committee for Clinical Laboratory Standards. Performance standards for antimicrobial disk and diluition susceptibility tests for bacteria isolated from animals; approved standard - Second edition 2002; M31- A2. National Committee for Clinical Laboratory Standard, Wayne, Pa.

13. Sambrook J, Fritsch EF Maniatis T. Molecular cloning: a laboratory manual. $2^{\text {nd }}$ ed. Cold Spring Harbor Laboratory Press 1989; Cold Spring Harbor, NY

14. Schwarz S, Chaslus-Dancla E. Use of antimicrobials in veterinary medicine and mechanisms of resistance. Vet Res 2001; 32: 201-225

15. Shaw KJ, Rather PN, Hare RS, Miller GH. Molecular genetics of aminoglycoside resistence genes and familial relationships of the aminoglycoside-modifing enzymes. Microbiolgical Reviews1993; 57(1): 138-163

16. Southern EM. Detection of specific sequences among DNA fragments separated by gel electrophoresis. Mol Biol 1975; 98: 503-517

17. Sundin GW. Distinct recent lineages of the $\operatorname{str} A-\operatorname{str} B$ streptomycin-resistance genes in clinical and enviromental bacteria. Current microbiol 2002; 45: 63-9

18. Sundin GW, Bender CL. Dissemination of the $\operatorname{str} A$ $s t r B$ streptomycin-resistance genes among commensal and pathogenic bacteria from humans, animals and plants. Mol Ecol 1996a; 5(1): 133-143

19. Threlfall EJ. Antimicrobial drug resistance in Salmonella: problems and perspectives in food- and water-borne infection. FEMS Microbiol Rev Jun 2002; 26 (2): 141-8

20. Threlfall EJ, Fisher IS, Berghold C, et al. Antimicrobial drug resistance in isolates of Salmonella enterica from cases of salmonellosis in humans in Europe in 2000: results of international multi-centre surveillance. Euro Surveill. 2003; 8: 41-5.

Alessandra Carattoli
Istituto Superiore di Sanità
Dip.to di Malattie Infettive, Parassitarie ed
Immunomediate
Reparto di Malattie Gastroenteriche e
Neurologiche
Viale Regina Elena, 299 - 00161 - Roma
Tel.: 06-49903128 - Fax: 06-49387112
E-mail: alecara@iss.it

